\title{
An Improved Demodulation for LoRa Through a Threshold- Based Detection of Error
}

This paper was downloaded from TechRxiv (https://www.techrxiv.org).

LICENSE

CC BY 4.0

SUBMISSION DATE / POSTED DATE

29-06-2021 / 23-08-2021

CITATION

Savaux, Vincent (2021): An Improved Demodulation for LoRa Through a Threshold-Based Detection of Error. TechRxiv. Preprint. https://doi.org/10.36227/techrxiv.14877078.v2

$\mathrm{DOI}$

10.36227/techrxiv.14877078.v2 


\title{
An Improved Demodulation for LoRa Through a Threshold-Based Detection of Error
}

\author{
Vincent Savaux, Member, IEEE
}

\begin{abstract}
This paper deals with an improved demodulation technique for LoRa signal. The principle consists in detecting the possible errors of demodulation, by comparing the highest peak of the periodogram of the dechirped received signal weighted by a coefficient $\beta$ smaller than one with the other peaks of the periodogram. If an error is detected, a second demodulation stage is processed at an oversampling rate (OSR) higher than the first demodulation stage. Otherwise, the result of the first demodulation is kept. A thorough performance analysis of the suggested technique, based on order statistics, is carried out, in terms of detection rate, error rate, and complexity. It is shown that $\beta$ can be tuned to adjusts the trade-off between performance and complexity. Moreover, simulations results shows that it is possible, for relatively low signal-to-noise (SNR) values, to reach the performance of the demodulation at highest OSR while keeping the complexity of the demodulation at lowest OSR.
\end{abstract}

Index Terms—LoRa, Demodulation, Detection, Order Statistics

\section{INTRODUCTION}

In the recent years, the Internet of things (IoT), and particularly the low power wide area (LPWA) technologies, have enabled the connectivity of a constantly growing number of devices, offering digital transformation across industry verticals [1]. The LPWA solutions are able to achieve long range communications at low data rates, using low cost devices with long battery life. Among the LPWA technologies [2], [3], LoRa is one of the most deployed and studied, mainly because it allows the deployment of networks in non-licensed bands [4]. The LoRa physical layer (PHY) has been developed by Semtech, based on the signal modulation called chirp spread spectrum (CSS) originally described in [5].

Lots of aspects of the performance of the LoRa PHY has been extensively studied in the scientific literature. The overall properties of CSS modulation have been analyzed in [6], [7]. Furthermore, signal processing algorithms have been suggested for time and frequency synchronization [8]-[13], multiple demodulations in presence of collision of LoRa signals [14], or multipath channel estimation [15]. Otherwise, numerous papers deal with performance analysis of the LoRa CSS waveform. In [16]-[20] the symbol and bit error rates (SER and BER) of LoRa signal have been analytically derived or approximated considering different channel models. These results have been extended by the authors of [21], [22] to consider the overall frame error rate (FER) instead of the BER. Alternatively, the impact of the interference from other signals on the BER performance is investigated in [23].

Interestingly, some papers deal with the improvement of the LoRa demodulation through software define radio implementations [24] or cloud radio access network approach [25]. However,

\footnotetext{
Vincent Savaux is with Institute of Research and Technology $b<>$ com Rennes, France. email: \{vincent.savaux@b-com.com\}

Manuscript received 2021
}

to the best of the author's knowledge, there is no dedicated paper dealing with signal processing algorithms for the improvement of the LoRa demodulation specifically (in term of error rate). The main reason is that the usual LoRa demodulation is already designed to be optimal in the maximum likelihood (ML) sense. To improve the performance of the LoRa demodulation, a basic method then consists in considering an oversampled received signal to increase the signal-to-noise ratio (SNR). However, this signal processing technique is straightforward (i.e. without scientific contribution), and increases the complexity of the receiver.

In this paper, a new LoRa demodulation improving the error rate performance is suggested. The principle consists in three steps: i) the usual LoRa demodulation is performed, ii) a test allowing to detect possible errors of demodulation is then processed, iii) if an error is detected, a second demodulation at higher oversampling rate (OSR) than the first one is carried out, otherwise the result of the first one is kept. The decision rule for the test considers that an error of demodulation occurs if at least one peak of the periodogram of the demodulated LoRa symbol is higher than a threshold defined as the highest peak of the periodogram weighted by a coefficient $\beta \in[0,1]$. It results that, according to the chosen $\beta$ value, the suggested demodulation can approach the performance of the demodulation at highest OSR (third step) with a reduced complexity, even down to that of the usual LoRa demodulation (first step). Thus, the coefficient $\beta$ allows the receiver to adjust the trade-off between performance and complexity.

Beyond the simple idea of the proposed threshold-based demodulation, a thorough theoretical performance analysis of the method is developed based on order statistics, leading to closed-form expressions of the detection rate, the overall error rate, and the complexity. Moreover, the developments are validated through simulations results, which also show that the suggested technique is able to outperform the usual LoRa demodulation by several decibels for a similar asymptotical complexity. In addition, the impact of imperfect synchronization is discussed, it is also mentioned that the method could be easily adapted to other technologies, and otherwise the analysis could have an application in applied mathematics since it could lead to closed-form solutions of specific functions.

The rest of the paper is organized as follows: Section II introduces the basics on LoRa demodulation and the corresponding performance. The suggested threshold-based demodulation is described in Section III, and the performance analysis is developed in Section IV. Section V is dedicated to the simulations results, and the method is discussed in Section VI, which also introduces other applications. Finally, Section VII concludes this paper.

Notations: The normal font $x$ is used for scalars, and the lower-case and the upper-case $x$ and $X$ indicates samples in time and frequency domains, respectively. The probability of an event 
$E$ is denoted by $\mathbb{P}(E)$, and $\mathbb{E}\{$.$\} is the mathematical expectation.$ We define the order statistics as follows (we use the usual notations as in [26]): let $X_{0}, X_{1}, \ldots, X_{N-1}$ be $N$ random variables, then we denote by $X_{(0)} \leq X_{(1)} \leq . . \leq X_{(N-1)}$ the corresponding order statistics, i.e. $X_{(0)}$ is the smallest variable of the $X_{k}$, $k=0,1, N-1, X_{(1)}$ is the smallest variable larger than $X_{(0)}$, and so on such that $X_{(N-1)}$ is the largest variable of the $X_{k}$.

\section{LoRa System Model AND Performance}

This section presents the basics on LoRa modulation and demodulation, including the complexity analysis of the demodulation process. Furthermore, the analytical SER and BER expressions are provided as well.

\section{A. LoRa Modulation and Demodulation}

The modulation of the data symbols in LoRa is based on CSS modulation. The process can be summarized as follows: the binary data stream to be transmitted is split into packets of size $S F$, where $S F$ is called the spreading factor (SF). Then, the binary packet is mapped onto a symbol $s^{(m)}$ of duration $T_{s}$, where $m \in\left\{0,1, . ., 2^{S F}-1\right\}$ is the symbol index. Thus, the $\mathrm{SF}$ is a key parameter as it allows to adapt the LoRa symbol length and therefore its robustness against distortions, to the cost of a data rate loss, through the relation $B_{w} T_{s}=N=2^{S F}$ where $B_{w}$ is the signal bandwidth (typically $125 \mathrm{kHz}, 250 \mathrm{kHz}$, or $500 \mathrm{kHz}$ ). We define $r$ the OSR of the signal ( $r=1$ means Nyquist rate), then for any $n=0,1, . ., N^{\prime}-1$ where $N^{\prime}=r N$, and for any $S F$ value, the modulated LoRa symbol $s^{(m)}$ can be expressed by generalizing [7]-(13) as

$$
s^{(m)}[n]=\exp \left(2 j \pi \frac{n}{r}\left(\frac{n}{2 N^{\prime}}-\frac{1}{2}+\frac{m}{N}-u\left(\frac{n}{r}-N+m\right)\right)\right),
$$

where $u($.$) indicates the Heaviside step function.$

We assume that the LoRa signal is correctly synchronized at the receiver side [9]-[12], and then the residual synchronization errors can be neglected. Moreover, the LoRa signal bandwidth is narrow enough to consider a one-tap block fading channel $h$. Therefore, for any $n=0,1, . . N^{\prime}-1$, the received signal $r[n]$ is given by

$$
r[n]=h s^{(m)}[n]+w[n]
$$

where $w[n], n=0,1, . ., N^{\prime}-1$, are the independent and identically distributed (iid) samples of the complex additive white Gaussian noise (AWGN) such that $w[n] \sim \mathbb{C N}\left(0, \sigma^{2}\right)$. It must be noticed that $h$ can be a random variable, according to the channel model. Thus in the following, we consider both AWGN (where $h=1$ ) and Rayleigh channel models. In any case, we generally define the signal-to-noise ratio ( $\mathrm{SNR})$ as

$$
S N R=\frac{\mathbb{E}\left\{\left|h s^{(m)}[n]\right|^{2}\right\}}{\mathbb{E}\left\{|w[n]|^{2}\right\}}=\frac{\mathbb{E}\left\{|h|^{2}\right\}}{\sigma^{2}}
$$

1) LoRa Demodulation for OSR $r=1$ : The aim of the demodulation process in LoRa is to estimate the symbol index $m$ from the observation $r[n]$. To this end, a maximum likelihood (ML) estimator is optimal in condition of synchronized reception. The basic principle of the LoRa demodulation at OSR $r=1$, detailed in [10], [11], [16] for instance, consists in three steps. First, the quadratic component of phase of the LoRa symbol in (1) is removed through the "dechirp" operation:

$$
y[n]=r[n] \exp \left(-2 j \pi n\left(\frac{n}{2 N}-\frac{1}{2}\right)\right) .
$$

Then, a discrete Fourier transform (DFT) is applied:

$$
Y[k]=\frac{1}{\sqrt{N}} \sum_{n=0}^{N-1} y[n] e^{\frac{-2 j \pi n k}{N}}
$$

where $k=0,1, . ., N-1$. It is noteworthy that $Y[k]=\sqrt{N} \delta(k-$ $m)+W[k]$ when $k=m$, and $Y[k]=W[k]$ when $k \neq m$, where $\delta(k)$ is the Dirac impulse and $W[k]$ are the noise samples in frequency domain. Moreover, the noise samples $W[k]$ are all iid and $W[k] \sim \mathbb{C N}\left(0, \sigma^{2}\right)$. Finally, the ML estimator of $m$ yields

$$
\hat{m}=\underset{k \in[[0, N]]}{\arg \max }|Y[k]|^{2},
$$

where $|Y[k]|^{2}$ is called the periodogram of $y[n]$. By limiting the complexity analysis to the number of complex multiplications, we deduce that (4), (5), and (6) require $N, N \log (N)$, and $N$ operations, respectively.

2) LoRa Demodulation for OSR $r>1$ : The LoRa demodulation process for OSR $r>1$ is similar to (4)-(6) with some adaptations. The "dechirp" operation becomes:

$$
y[n]=r[n] \exp \left(-2 j \pi \frac{n}{r}\left(\frac{n}{2 N^{\prime}}-\frac{1}{2}\right)\right),
$$

where $n=0,1, . ., N^{\prime}$. Then, a DFT like (5) is applied, with size $N^{\prime}$ instead of $N$. However, unlike previously where the periodogram $|Y[k]|^{2}$ using $r=1$ yields one unique peak (in absence of noise) at the position $k=m$ and with the amplitude $N$, the periodogram $|Y[k]|^{2}$ using $r>1$ highlights two peaks at the positions $k=m$ and $k=m-N+N^{\prime}$, and with amplitudes $\frac{r(N-m)}{\sqrt{N^{\prime}}}$ and $\frac{r m}{\sqrt{N^{\prime}}}$, respectively. This property is proved in Appendix A, and shown in Fig. 1, where $r=2, N=128$, and $m=71$.

It should be noted from the previous results that the two peaks of interest are located in the index ranges $k \in[[0, N-1]]$ and $k \in\left[\left[N^{\prime}-N, N^{\prime}-1\right]\right]$, respectively. Therefore, the optimal way (in the ML sense) to combine the energy of both peaks consists in the following sum, for $k=0,1, \ldots, N-1$ :

$$
\tilde{Y}[k]=Y[k]+Y[(r-1) N+k] .
$$

It must be noticed that $\tilde{Y}[k]=\sqrt{N^{\prime}} \delta(k-m)+\tilde{W}[k]$ when $k=m$, and $\tilde{Y}[k]=\tilde{W}[k]$ when $k \neq m$, where $\tilde{W}[k]=W[k]+$ $W[(r-1) N+k]$ are the combined noise samples in frequency domain, which are all iid and $\tilde{W}[k] \sim \mathbb{C} \mathcal{N}\left(0,2 \sigma^{2}\right)$. Thus, it is worth noting that the ratio between the square amplitude of the Dirac pulse and the noise power depends on the OSR value $r$.

Finally, the estimation of $m$ is performed similarly to (6) where $Y[k]$ is substituted by $\tilde{Y}[k]$, with $k \in[[0, N]]$. Furthermore, (7), 


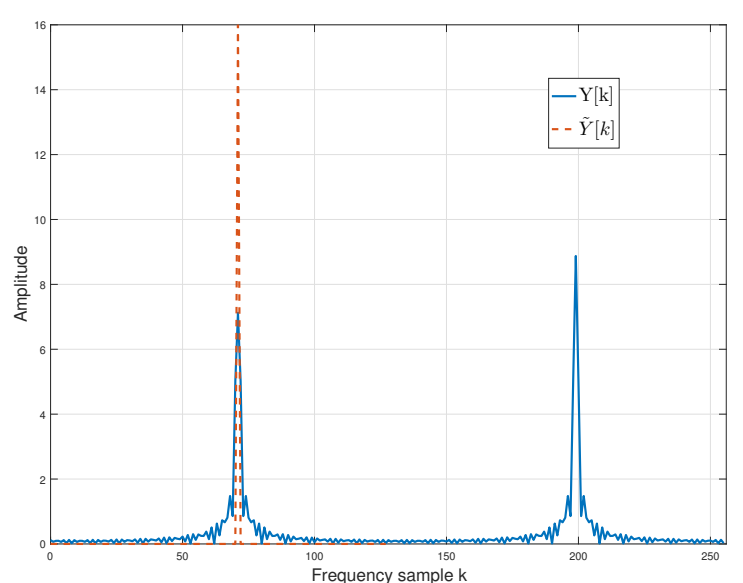

Fig. 1. Amplitude of $Y[k]$ and $\tilde{Y}[k]$ versus frequency samples $k$, using $r=2$, $N=128$, and $m=71$.

(5) (with $r>1$ ), and (6) (with $r>1$ ) requires $N^{\prime}, N^{\prime} \log \left(N^{\prime}\right)$, and $N$ operations, respectively. However, the complexity of the DFT can be substantially reduced by noticing that the $N^{\prime}-2 N$ samples in the range $k \in\left[\left[N, N^{\prime}-N-1\right]\right]$ are unnecessary in the demodulation process for $r>1$, and their computation can then be avoided. As a consequence, the complexity of the DFT can be reduced to $2 N \log \left(N^{\prime}\right)$ and even $2 N \log (2 N)$ in practice [27], since the DFT is deterministic. In the following, we provide the analytical expressions of the achievable SER and BER for any OSR, since it will be used in the further developments.

\section{B. SER and BER Performance}

The symbol error rate of LoRa modulation, given the channel gain $h$, is defined as $P_{s \mid h}=\mathbb{P}(\hat{m} \neq m)$. It is convenient to develop $\mathbb{P}(\hat{m} \neq m)$ by using the order statistics. Thus, let us define $X_{k}=|Y[k]|^{2}$, then $X_{k}$ obeys a $\chi^{2}$ distribution if $k \neq m$ and a non-central $\chi^{2}$ distribution if $k=m$. Moreover, we denote by

$$
X_{(0)} \leq . . \leq X_{(k)} \leq . . \leq X_{(N-2)}
$$

the $N-1$ ordered random variables obeying a $\chi^{2}$ distribution, i.e. any $X_{(k)} \neq X_{m}$. Then it results that we can rewrite $P_{s \mid h}$ as

$$
\begin{aligned}
P_{s \mid h} & =\mathbb{P}(\hat{m} \neq m) \\
& =\mathbb{P}\left(X_{(N-2)} \geq X_{m}\right),
\end{aligned}
$$

highlighting that a symbol is erroneously estimated when at least the highest peak of the periodogram corresponding to noise only is larger that the peak containing the Dirac pulse of amplitude $N$. The SER expression of the LoRa signal sampled at Nyquist rate, and given the channel gain $h$, is well known from [17]-[19], and hereby reported:

$$
P_{s \mid h}=-\sum_{k=1}^{N-1} \frac{(-1)^{k}\left(\begin{array}{c}
N-1 \\
k
\end{array}\right)}{k+1} \exp \left(-\frac{k N|h|^{2}}{(k+1) \sigma^{2}}\right) .
$$

We here provide a more general SER expression for any OSR $r>1$, and given the suggested demodulation for $r>1$ as follows:

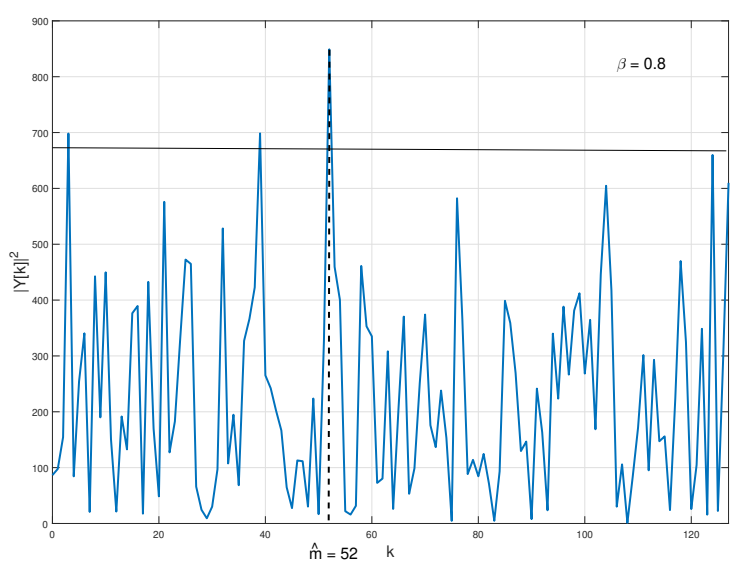

Fig. 2. Illustration of the principle of the detector of errors of demodulation, where $\beta=0.8, \hat{m}=52$, and $\Omega_{k, \beta}=\{3,39\}$.

$$
P_{s \mid h}=-\sum_{k=1}^{N-1} \frac{(-1)^{k}\left(\begin{array}{c}
N-1 \\
k
\end{array}\right)}{k+1} \exp \left(-\frac{k N^{\prime}|h|^{2}}{2(k+1) \sigma^{2}}\right),
$$

where $N$ has been substituted by $N^{\prime}$ and $\sigma^{2}$ by $2 \sigma^{2}$ in the exponential. This highlights that, for any oversampling rate $r>1$, the amplitude of the Dirac pulse in $Y[m]$ is $\sqrt{N^{\prime}}$, while the symbol index $m$ is estimated within the interval $[[0, N]]$ in (6). Moreover, the factor $2 \sigma^{2}$ comes from the sum of noise components $\tilde{W}[k]=W[k]+W[(r-1) N+k]$ in (8). Different theoretical SER expressions have been developed in [19] from (11) with $r=1$ according to the channel model, such as AWGN, Rayleigh, Rice, or Nakagami, and can be straightforwardly extended to the more general expression (11) where $r>1$. The BER, in turn, is simply expressed as $P_{e}=\frac{2^{S F-1}}{2^{S F}-1} P_{s}$.

We deduce from (11) that the larger the value $N^{\prime}$ (i.e. the OSR $r$ ) the lower the SER and BER. A simple method to improve the performance of the LoRa demodulation then consists in increasing the OSR at the receiver side. However, a performance improvement is only effective for $r>2$, as $r=2$ in (11) leads to the same SER as in (10) using $r=1$. Furthermore, the performance improvement by means of an increase of the OSR is obtained to the cost of a non-linear increase of the complexity, which is the main limit of this solution. We hereby suggest an alternative low-complexity demodulation technique based on the detection of possible symbol errors at OSR $r=1$.

\section{Threshold-BASED Demodulation}

In this section, we describe the suggested demodulation based on the detection of symbol errors. Moreover, we define notations that will be used in the performance analysis.

\section{A. Error Detection}

The basic idea of the proposed improved demodulation is to perform the usual LoRa symbol demodulation (4)-(6) at OSR $r_{1}$ (typically $r_{1}=1$ ), and to check if it is likely that the symbol $m$ 
has been erroneously estimated. If an error is detected, then a second demodulation at OSR $r_{2}>r_{1}$ is performed, otherwise the receiver keeps the first estimated $\hat{m}$ value obtained at OSR $r_{1}$. The following decision rule for the error detection is arbitrarily considered for its simplicity: a coefficient $\beta \in[0,1]$ is set such that a possible symbol error is decided if it exists $k \in[[0, N]]$ such that:

$$
|Y[k]|^{2} \geq \beta|Y[\hat{m}]|^{2}, \quad k \neq \hat{m} .
$$

where $|Y[\hat{m}]|^{2}$ is by definition in (6) the largest peak of the periodogram. This event is illustrated in Fig. 2, where $\beta=0.8$, and two peaks exceeds $\beta|Y[\hat{m}]|^{2}$. Accordingly, we define $\Omega_{k, \beta}$ the set of indexes $k$ such that (12) is true. Thus, in Fig. 2, we can observe that $\Omega_{k, \beta}=\{3,39\}$. The suggested demodulation can be summarized by three main steps:

1) Estimate $\hat{m}$ through the LoRa symbol demodulation (4)-(6) at OSR $r_{1}$.

2) Perform the test (12). If $\Omega_{k, \beta}=\emptyset$, then the demodulation is finished, otherwise $\left(\Omega_{k, \beta} \neq \emptyset\right)$ go to step 3 .

3) Estimate again $\hat{m}$ through the LoRa symbol demodulation in Section II-A2 at OSR $r_{2}>r_{1}$.

Intuitively, we understand that the use of the demodulation at OSR $r_{2}>r_{1}$ shall improve the performance, whereas the test in step 2 shall limit the complexity, in particular since the increase of complexity is negligible ((12) only involves one multiplication and $N-1$ comparisons). In addition, it is unlikely for the test (12) to be true when $\sigma^{2}$ is weak compared with $N^{\prime}$, i.e. in moderately high SNR range. These remarks will be theoretically analyzed in Section IV, and numerically illustrated in Section V. Prior to this, we hereby introduce some definitions and notations in order to ease and clarify the further developments.

\section{B. Definitions and Notations}

Definition 1. We define $E$ as the following event: $\exists k$, $k=0,1, \ldots, N-1$, such that $k \neq \hat{m}$, and $\beta \in[0,1]$, such that $|Y[k]|^{2} \geq \beta|Y[\hat{m}]|^{2}$. Thus, $E$ corresponds to the event "an error is detected".

Definition 2. From the definition of $E$, we also define the sub-events $E_{1}$ and $E_{0}$ as follows:

- $E_{1}$ is similar to the event $E$ (an error is detected), conditionally to $\hat{m}=m$, i.e. the symbol $m$ is properly estimated;

- $E_{0}$ is similar to the event $E$ (an error is detected), conditionally to $\hat{m} \neq m$, i.e. the symbol $m$ is erroneously estimated.

Thus, by referring to the usual terms used in detection theory, $E_{1}$ can be seen as the "false alarm" event, whereas $E_{0}$ is rather the "good detection" event. In turn, the probability of $E$ can be defined as the overall detection rate. From Definitions 1 and 2, we deduce that, given $h$, the mathematical relationship between the probability of the event $E$ and that of both events $E_{1}$ and $E_{0}$ is

$$
\begin{aligned}
P_{E \mid h} & =\mathbb{P}(E \cap \hat{m}=m)+\mathbb{P}(E \cap \hat{m} \neq m) \\
& =P_{E_{1} \mid h}\left(1-P_{s \mid h}\right)+P_{E_{0} \mid h} P_{s \mid h} .
\end{aligned}
$$

It is worth to be claimed that, unlike usual detection theory where it is sought to maximize $P_{E_{1} \mid h}$ while minimizing $P_{E_{0} \mid h}$, we here consider the test in (12) for its simplicity, its inherent performance being evaluated in Sections IV and V.

\section{Performance Analysis}

In this section, we derive the detection rate of the demodulation error detector, and then we provide the performance analysis of the suggested demodulation method in terms of SER/BER and complexity. Both AWGN and Rayleigh channel models are considered. To simplify the developments, we suppose that the first demodulation of the suggested method is performed at $r_{1}=1$, but the results remain valid for $r_{1}>1$ by substituting $N$ by $N^{\prime}$ and $\sigma^{2}$ by $2 \sigma^{2}$ within the sums.

\section{A. Detection Rate}

The detection rate $P_{E \mid h}$ requires the assessment of $P_{E_{1} \mid h}$ and $P_{E_{0} \mid h}$, such as given in Propositions 1 and 2. To this end, and for a clarity matter, we keep the same notations as previously defined in Section II to obtain (11), i.e. $X_{k}=|Y[k]|^{2}$, and $X_{(k)}$ the corresponding ordered variables.

Proposition 1. For any $N=2^{S F}, \beta \in[0,1]$, and given any channel channel coefficient $h \in \mathbb{C}$, the probability $P_{E_{1} \mid h}$ (i.e. the probability of the event $E_{1}$ ) is given by:

$$
\begin{aligned}
P_{E_{1} \mid h} & =\mathbb{P}\left(X_{(N-2)} \geq \beta X_{\hat{m}} \mid \hat{m}=m\right) \\
& =\mathbb{P}\left(X_{(N-2)} \geq \beta X_{m} \mid X_{m} \geq X_{(N-2)}\right),
\end{aligned}
$$

where $X_{(N-2)}$ satisfies $X_{(N-2)} \neq X_{m}$ and is therefore equal to one of the $|Y[k]|^{2}$ obeying a $\chi^{2}$ distribution. The development of (14) leads to the closed-form expression (15).

Proof. See Appendix B for the proof of $P_{E_{1} \mid h}$ such as expressed in (15).

Proposition 2. For any $N=2^{S F}, \beta \in[0,1]$, and given any channel channel coefficient $h \in \mathbb{C}$, the probabilities $P_{E_{0} \mid h}$ (i.e. the probability of the event $E_{0}$ ) is given by:

$$
\begin{aligned}
P_{E_{0} \mid h} & =\mathbb{P}\left(X_{(N-2)} \geq \beta X_{\hat{m}} \mid \hat{m} \neq m\right) \\
& =\mathbb{P}\left(X_{(N-2)} \geq \beta X_{\hat{m}}|| X_{\hat{m}} \geq X_{m}\right),
\end{aligned}
$$

where $X_{(N-2)}$ is either a variable obeying a $\chi^{2}$ distribution (if $X_{(N-2)} \neq|Y[m]|^{2}$ ), or a variable obeying a non-central $\chi^{2}$ distribution (if $X_{(N-2)}=|Y[m]|^{2}$ ). The development of (16) leads to the closed-form expression (17).

Proof. See Appendix $\mathrm{C}$ for the proofs of $P_{E_{0} \mid h}$ such as expressed in (17).

It can be directly verified from (14) and (15) that $P_{E_{1} \mid h}(\beta=$ $0)=1$ and $P_{E_{1} \mid h}(\beta=1)=0$, as well as from (16) and (17) we have $P_{E_{0} \mid h}(\beta=0)=1$ (this result is a bit more tricky to prove, but not shown in this paper) and $P_{E_{0} \mid h}(\beta=1)=0$. In turn, we deduce from (13) that $P_{E \mid h}(\beta=0)=1$ and $P_{E \mid h}(\beta=1)=0$. This is consistent with the test in (12) since all peaks $|Y[k]|^{2}, k \neq \hat{m}$ of the periodogram respect the inequality $|Y[k]|^{2} \geq 0(\beta=0)$ and no peak respects the inequality $|Y[k]|^{2} \geq|Y[\hat{m}]|^{2}(\beta=1)$. Moreover, from (13), (15), and (17), we deduce that the overall detection rate in AWGN channel, denoted by $P_{E}^{A W G N}$, is given by

$$
P_{E}^{A W G N}=P_{E \mid h=1},
$$




$$
P_{E_{1} \mid h}=\frac{1}{1-P_{s \mid h}} \sum_{k=0}^{N-1}(-1)^{k}\left(\begin{array}{c}
N-1 \\
k
\end{array}\right)\left(\frac{\exp \left(-\frac{k N|h|^{2}}{(k+1) \sigma^{2}}\right)}{k+1}-\frac{\exp \left(-\frac{\beta k N|h|^{2}}{(\beta k+1) \sigma^{2}}\right)}{\beta k+1}\right)
$$

$$
\begin{aligned}
P_{E_{0} \mid h}= & \frac{(N-1)(N-2)}{P_{s \mid h}}\left[\frac{1}{N-2}\left(\frac{1}{2} \exp \left(-\frac{N|h|^{2}}{2 \sigma^{2}}\right)-\frac{\beta}{1+\beta} \exp \left(-\frac{N|h|^{2}}{(\beta+1) \sigma^{2}}\right)\right)\right. \\
& \left.-\sum_{k=0}^{N-3}(-1)^{k}\left(\begin{array}{c}
N-3 \\
k
\end{array}\right)\left(\frac{\exp \left(-\frac{(k+2) N|h|^{2}}{(k+3) \sigma^{2}}\right)}{(k+1)(k+2)(k+3)}-\frac{\exp \left(-\frac{\left(k+1+\frac{1}{\beta}\right) N|h|^{2}}{\left(k+2+\frac{1}{\beta}\right) \sigma^{2}}\right)}{\beta(k+1)\left(k+1+\frac{1}{\beta}\right)\left(k+2+\frac{1}{\beta}\right)}\right)\right] .
\end{aligned}
$$

where $h=1$ is substituted in (15) and (17). If a Rayleigh channel is assumed, then $|h|^{2}$ obeys a $\chi^{2}$ distribution with two degrees of freedom, which is defined as

$$
f_{|h|^{2}}(x)=\frac{e^{-\frac{x}{\sigma_{h}^{2}}}}{\sigma_{h}^{2}}
$$

where $\sigma_{h}^{2}=\mathbb{E}\left\{|h|^{2}\right\}$ is the variance of the channel coefficient, and $x \in \mathbb{R}_{+}$. The detection rate in Rayleigh channel, expressed as $P_{E}^{R a y}$, is obtained by

$$
\begin{aligned}
P_{E}^{\text {Ray }}= & \int_{0}^{+\infty} P_{E \mid h} f_{|h|^{2}}(x) d x \\
= & \int_{0}^{+\infty} P_{E_{1} \mid h}\left(1-P_{s \mid h}\right) f_{|h|^{2}}(x) d x \\
& +\int_{0}^{+\infty} P_{E_{0} \mid h} P_{s \mid h} f_{|h|^{2}}(x) d x,
\end{aligned}
$$

where $|h|^{2}=x$ is substituted in the expressions of $P_{E_{1} \mid h}$ and $P_{E_{0} \mid h}$ in (15) and (17). Thus, the first integral in the right side of (20) leads to

$$
\begin{aligned}
& \int_{0}^{+\infty} P_{E_{1} \mid h}\left(1-P_{s \mid h}\right) f_{|h|^{2}}(x) d x \\
& =\sum_{k=0}^{N-1}(-1)^{k}\left(\begin{array}{c}
N-1 \\
k
\end{array}\right) \\
& \times\left(\frac{\sigma^{2}}{k N \sigma_{h}^{2}+(k+1) \sigma^{2}}-\frac{\sigma^{2}}{\beta k N \sigma_{h}^{2}+(\beta k+1) \sigma^{2}}\right),
\end{aligned}
$$

and the second one, $\int_{0}^{+\infty} P_{E_{0} \mid h} P_{s \mid h} f_{|h|^{2}}(x) d x$ leads to (22).

The formulas (13)-(22) allow us to derive the analytical closed-form expressions of the detection rate in AWGN and Rayleigh channel models. Despite their apparent complexity, it is worth noticing that, asymptotically, $P_{E}^{A W G N}$ and $P_{E}^{R a y}$ tends to 0 when $\sigma^{2}$ tends to 0 (or equivalently when the SNR tends to $+\infty$ ). It highlights that it is unlikely that a peak of the periodogram becomes larger than $\beta|Y[m]|^{2}$ for a sufficiently high SNR. The SNR range of validity of this remark will be illustrated through numerical results in Section V. Beforehand, we use the previous developments of the detection rate to derive the analytical expressions of the achievable SER/BER error rate and the complexity of the suggested demodulation.

\section{B. SER/BER Performance of the Suggested Demodulation Method}

For clarity purpose, we hereby highlight the OSR by the superscript $(r)$. Thus for instance, $P_{s \mid h}^{(1)}$ is the symbol error rate for OSR1. Let us consider two different OSRs $r_{1}$ and $r_{2}$ such as $r_{1}<r_{2}$ (typically $r_{1}=1$ and $r_{2}>2$ ), then the overall SER performance of the suggested demodulation method, given $h$, denoted by $P_{s, N D \mid h}$, involving a first demodulation at OSR $r_{1}$, and the second one at OSR $r_{2}$, can be expressed as

$$
P_{s, N D \mid h}=P_{s \mid h}^{\left(r_{1}\right)}\left(1-P_{E_{0} \mid h}^{\left(r_{1}\right)}\right)+P_{E \mid h}^{\left(r_{1}\right)} P_{s \mid h}^{\left(r_{2}\right)}
$$

where $P_{s \mid h}^{\left(r_{1}\right)}\left(1-P_{E_{0} \mid h}\right)$ is the probability that an error occurs at OSR $r_{1}$ and is not detected, and $P_{E \mid h} P_{s \mid h}^{\left(r_{2}\right)}$ is the the probability that an error occurs at OSR $r_{2}$ while an error has been previously detected. The overall SER performance $P_{s, N D}$ then depends on the channel model, and can be expressed and calculated in closed form in a similar way as in (18) and (20) when AWGN and Rayleigh channel model is considered, respectively.

It is worth noticing from (23) and from what has been previously proved that, for any $\sigma^{2}$ value, we have the following bounds for $P_{s, N D \mid h}$ :

$$
\begin{array}{rlrl}
P_{s, N D \mid h}(\beta=1) & \geq P_{s, N D \mid h} \geq P_{s, N D \mid h}(\beta=0) \\
\Leftrightarrow \quad & P_{s \mid h}^{\left(r_{1}\right)} & \geq P_{s, N D \mid h} \geq P_{s \mid h}^{\left(r_{2}\right)} .
\end{array}
$$

This result confirms the intuitive fact that the demodulation method can be configured as a function of $\beta$ to reach any expected performance within the set $\left[P_{s \mid h}^{\left(r_{2}\right)}, P_{s \mid h}^{\left(r_{1}\right)}\right]$. This will further shown through numerical results in Section V.

\section{Complexity Analysis}

Once again, we hereby highlight the OSR by the superscript $(r)$, e.g. $N^{(r)}=r .2^{S F}$, and we suppose that $r_{1}<r_{2}$. The complexity analysis considers the number of complex multiplications. It consists in the demodulation at OSR $r_{1}$, and the demodulation at OSR $r_{2}$ when a possible error has been detected. Thus, the overall complexity, denoted by $C_{N D}$, of the suggested demodulation is given by

$$
\begin{aligned}
C_{N D}= & N+N^{\left(r_{1}\right)}\left(1+\log \left(N^{\left(r_{1}\right)}\right)\right) \\
& +P_{E}^{\left(r_{1}\right)}\left(N+N^{\left(r_{2}\right)}\left(1+\log \left(N^{\left(r_{2}\right)}\right)\right)\right),
\end{aligned}
$$




$$
\begin{aligned}
\int_{0}^{+\infty} P_{E_{0} \mid h} P_{s \mid h} f_{|h|^{2}}(x) d x= & \frac{N-1}{N-2}\left(\frac{\sigma^{2}}{N \sigma_{h}^{2}+2 \sigma^{2}}-\frac{\beta \sigma^{2}}{N \sigma_{h}^{2}+(\beta+1) \sigma^{2}}\right) \\
& -\sum_{k=0}^{N-3}(-1)^{k}\left(\begin{array}{c}
N-3 \\
k
\end{array}\right)\left(\frac{(N-1)(N-2) \sigma^{2}}{(k+1)(k+2)\left((k+2) N \sigma_{h}^{2}+(k+3) \sigma^{2}\right)}\right. \\
& \left.-\frac{(N-1)(N-2) \sigma^{2}}{\beta(k+1)\left(k+1+\frac{1}{\beta}\right)\left(\left(k+1+\frac{1}{\beta}\right) N \sigma_{h}^{2}+\left(k+2+\frac{1}{\beta}\right) \sigma^{2}\right)}\right) .
\end{aligned}
$$

where $P_{E}^{\left(r_{1}\right)}=P_{E}^{A W G N}$ or $P_{E}^{\left(r_{1}\right)}=P_{E}^{R a y}$ according to the considered channel model. Interestingly, it has been stated that $P_{E}$ tends to zero for high SNR values. We conclude that the complexity of the suggested method tends to that of the demodulation carried out at OSR $r_{1}$, i.e. the complexity of the suggested method decreases when the SNR increases. Further simulations results will be provided in Section V to numerically illustrate this property. More generally, from (24) and (25), we deduce that the threshold value $\beta$ should be chosen to optimize the trade-off between performance and complexity.

\section{Simulations Results}

The simulations results have been obtained using Matlab, and the theoretical results in (18) and (20) have been computed with Python 3 including numpy library for a sake of computation precision. Up to $10^{8}$ independent Monte-Carlo runs have been performed to attain a satisfactory precision in the simulated results. In all simulations a SF7 LoRa signal with $B_{w}=125$ $\mathrm{kHz}$ has been considered, but it must be mentioned that similar results are obtained for larger SF (curves are shifted to the left). Moreover, we set the oversampling rates to $r_{1}=1, r_{2}=4$, and the variance of the Rayleigh channel coefficient to $\sigma_{h}^{2}=1$.

Fig. 3 shows the detection rate $P_{E}, P_{E_{1}}$, and $P_{E_{0}}$ versus SNR (dB) in AWGN channel and $\beta=0.8$, for both the theoretical results and the simulated ones. It can be observed that the numerical results and the theoretical results match, therefore validating the developments (15) and (17) in Propositions 1 and 2. It can also be seen that $P_{E_{0}}$ increases and $P_{E_{1}}$ decreases to zero when the SNR increases. This means that, when the SNR increases, it is more likely to correctly detect the actual error while limiting the "false alarm" probability. In addition, the overall detection rate $P_{E}$ decreases to zero when the SNR increases, mainly due to the fact that the number of errors of demodulation tends to zero as the SNR increases.

In Fig. 4 is depicted the detection rate $P_{E}$ versus SNR (dB) in AWGN and Rayleigh channels for $\beta \in\{0.6,0.9\}$, obtained through simulations and through analysis (18) (AWGN) and (20) (Rayleigh). Once again, it can be observed that both theoretical and numerical results match for both $\beta$ values, therefore validating the theoretical developments.

Fig. 5 shows the SER performance of the suggested new demodulation (ND) versus SNR (dB) in AWGN channel (a) and Rayleigh channel (b), for different values of $\beta \in\{0.6,0.7,0.8,0.9\}$. The performance of the usual LoRa demodulation with OSR1 is also plotted as reference. It can be observed in Fig. 5-(a) that, at $\mathrm{SER}=10^{-2}$, a gain of $0.5 \mathrm{~dB}$

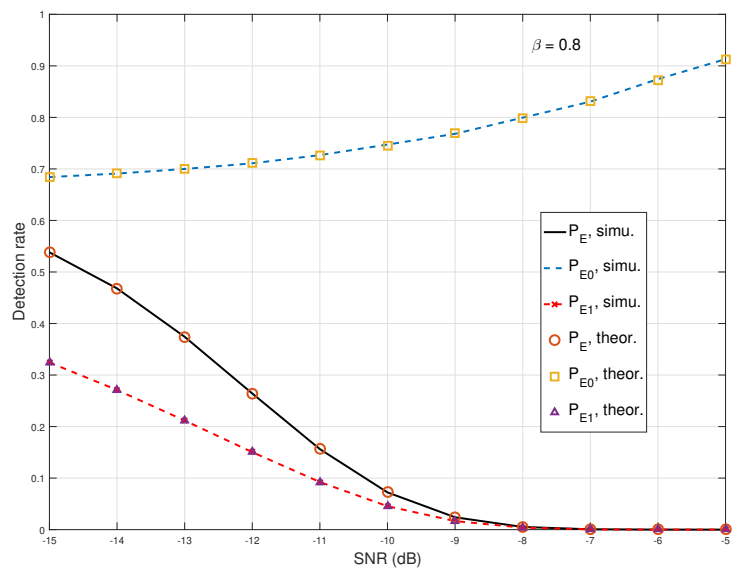

Fig. 3. $P_{E}, P_{E 1}$, and $P_{E 0}$ versus SNR (dB) in AWGN channel and $\beta=0.8$. Comparison of theoretical and numerical results.

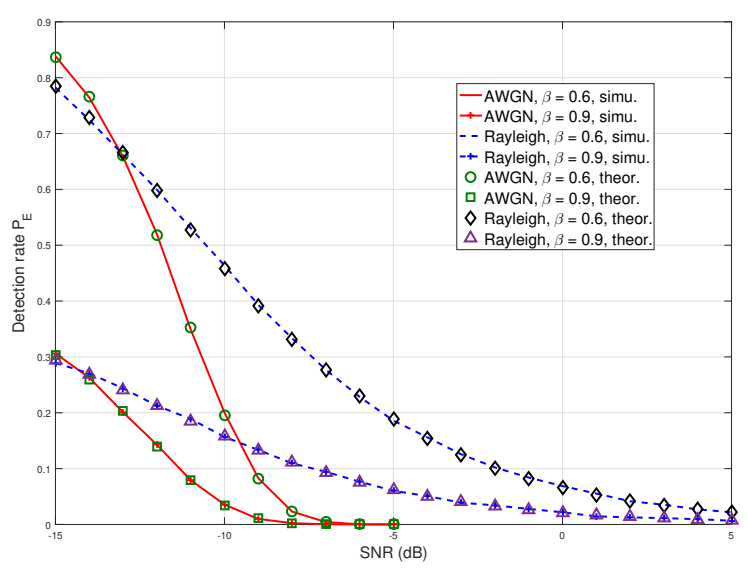

Fig. 4. Detection rate $P_{E}$ versus SNR (dB) in AWGN and Rayleigh channels for $\beta \in\{0.6,0.9\}$. Comparison of theoretical and numerical results.

is achieved by the suggested method for $\beta=0.9$ compared with OSR1, and up to $2.5 \mathrm{~dB}$ when $\beta=0.6$. This actually confirms that the lower $\beta$, the better the performance since the number of detected errors increases when $\beta$ decreases. This behavior can be observed as well in Rayleigh channel in Fig. 5-(b), where a gain of $1 \mathrm{~dB}$ is achieved by the suggest method for $\beta=0.9$ compared with OSR1, and up to $2.9 \mathrm{~dB}$ when $\beta=0.6$. These numerical results then validate the good performance of the suggested 


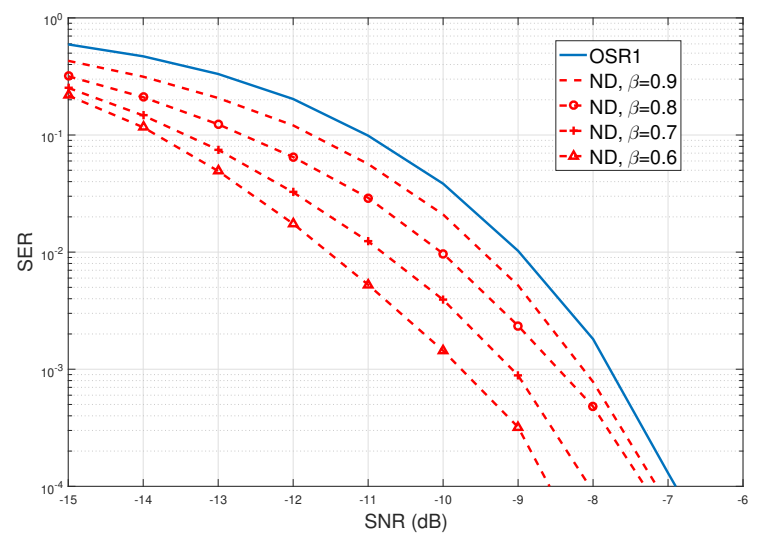

(a) AWGN channel.

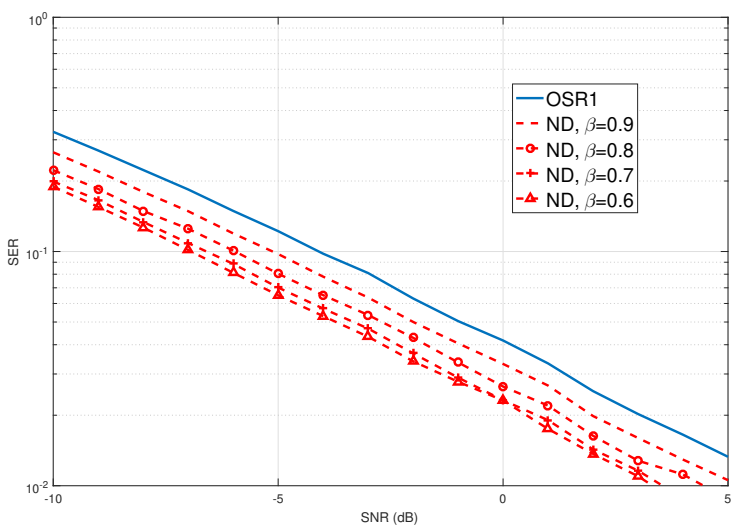

(b) Rayleigh channel.

Fig. 5. SER versus SNR (dB) of the usual LoRa demodulation at OSR1, compared with the suggested new demodulation (ND) for $\beta \in\{0.6,0.7,0.8,0.9\}$, in AWGN channel (a) and Rayleigh channel (b).

demodulation as it largely outperforms the demodulation at OSR1, and this performance can be configured through $\beta$.

To complete the performance results, Fig. 6 shows the complexity versus the SNR (dB), keeping the same configuration as in Fig. 5. It can be observed in both Figs. 6-(a) (AWGN) and (b) (Rayleigh) that for extremely low SNR values, the complexity of the suggested method increases since the detection rate increases. However, such as expected, we notice that the complexity tends to that of OSR1 when the SNR increases, even for relatively low SNR values. To give a numerical example from Figs.5 -(a) and 6-(a) (AWGN), at $\mathrm{SNR}=-10 \mathrm{~dB}$, we can observe that the SER of the new demodulation is decreased by a factor 2 (resp. 30) compared with OSR1, for a complexity increase of a factor 1.08 (resp. 1.47) for $\beta=0.9$ (resp. $\beta=0.6$ ). To give a more general remark, we can reasonably observe that a coefficient $\beta=0.7$ or 0.8 gives a good trade-off between the SER performance and the complexity for $\mathrm{SNR} \geq-10 \mathrm{~dB}$ in AWGN channel, and for $\mathrm{SNR} \geq-5 \mathrm{~dB}$ in Rayleigh channel.

\section{DISCUSSION}

It has been theoretically and numerically proved that the suggested demodulation method offers an excellent trade-off between

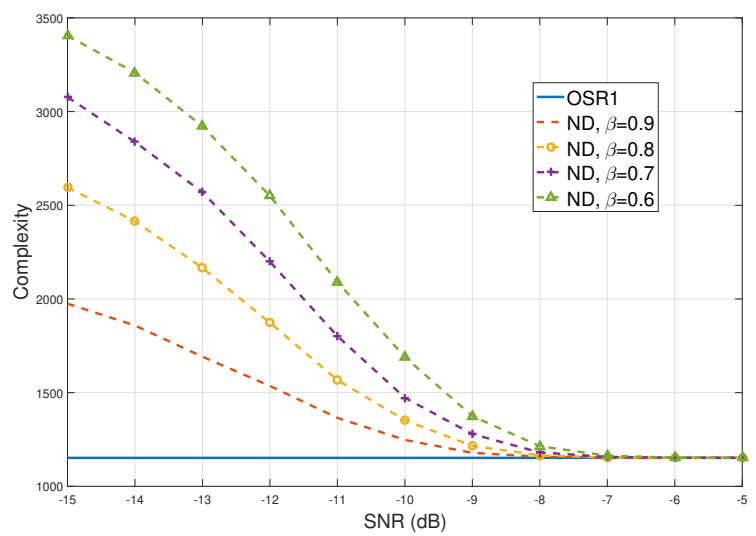

(a) AWGN channel.

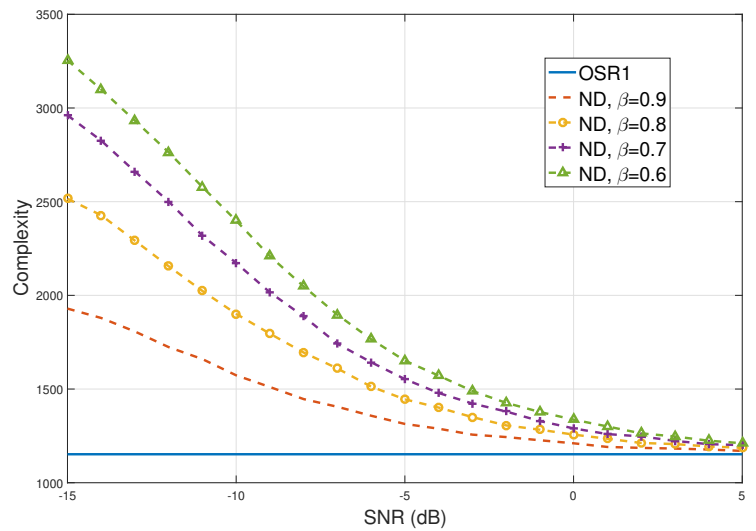

(b) Rayleigh channel.

Fig. 6. Complexity (25) versus SNR (dB) of the usual LoRa demodulation at OSR1, compared with the suggested new demodulation (ND) for $\beta \in\{0.6,0.7,0.8,0.9\}$, in AWGN channel (a) and Rayleigh channel (b).

performance and complexity. However, this has been carried out in good conditions where the residual synchronization errors have been neglected. To complete the above results, Fig. 7 shows the SER versus SNR (dB) of the suggested new demodulation (ND) as well as OSR1 for $\beta=0.7$, in AWGN channel considering a residual frequency offset. The previous results considering perfect conditions have been reported as well. The frequency offset has been randomly chosen within $\left[-\frac{B_{w}}{2 N}, \frac{B_{w}}{2 N}\right]$, where $\frac{B w}{2 N} \approx 488.28 \mathrm{~Hz}$ corresponds to half the distance between two frequency bin. It can observed that, in the considered SNR range, the presence of the frequency offset only involves a performance reduction, highlighted by a shift to the right. However, the same performance gain as in Fig. 5-(a) is achieved by the new demodulation compared with OSR1. This shows that an imperfect synchronization has a priori no effect on the detection error.

It is also interesting to notice that the basic principle of the proposed LoRa demodulation could be extended by repeating steps 2 and 3 once or until $\Omega_{k, \beta}=\emptyset$, to the cost of an increase of complexity. However, this would deserve a dedicated work. More interesting, the new demodulation could be used in other applications and technologies as soon as it involves demodulation of sinusoids or more generally constant modulus signals. For 


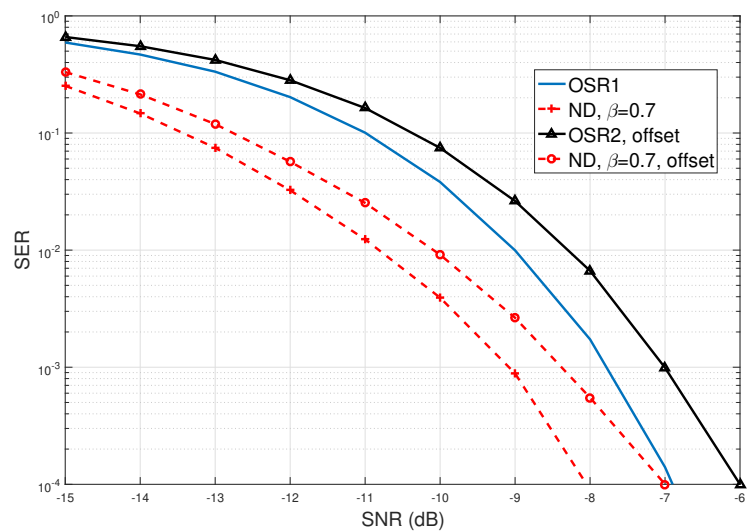

Fig. 7. SER versus SNR (dB) of the usual LoRa demodulation at OSR1, compared with the suggested new demodulation (ND) for $\beta=0.7$, in AWGN channel considering a residual frequency offset.

instance, one can cite the narrowband-IoT (NB-IoT) standard, where the suggested method could be easily adapted to be used for the estimation of the random access preamble identifier (RAPID), which is a mandatory parameter in the random access procedure in NB-IoT. In fact, the RAPID corresponds to the frequency position of the first transmitted sinusoid composing the preamble transmitted by the device to the evolved node B (eNB).

Another possible application of the present study would lead to closed-form expressions of some integrals involving the $\mathrm{Q}$ Marcum function [28]. Thus, for instance, by denoting $f_{Z}$ the non-central $\chi^{2}$ distribution function, then the numerator of (32) can be re-expressed as

$$
\begin{aligned}
& \mathbb{P}\left(X_{(N-2)} \geq \beta Z \cap Z \geq X_{(N-2)}\right) \\
= & \mathbb{P}\left(\frac{2 X_{(N-2)}}{\beta \sigma^{2}} \geq \frac{2 Z}{\sigma^{2}} \geq \frac{2 X_{(N-2)}}{\sigma^{2}}\right) \\
= & \int_{0}^{+\infty} f_{(N-1)}(x) \int_{\frac{2 x}{\sigma^{2}}}^{\frac{2 x}{\beta \sigma^{2}}} f_{Z}(z) d z d x \\
= & \int_{0}^{+\infty} \frac{(N-1)}{4}\left(1-\frac{e^{-\frac{x}{2}}}{2}\right) e^{-\frac{x}{2}} \\
& \times\left[Q_{1}\left(\sqrt{\lambda}, \sqrt{\frac{2 x}{\sigma^{2}}}\right)-Q_{1}\left(\sqrt{\lambda}, \sqrt{\frac{2 x}{\beta \sigma^{2}}}\right)\right] d x,
\end{aligned}
$$

where $\lambda=\frac{2|h|^{2}}{\sigma^{2}}$. It must be noted that the integral in (26) has a closed-form expression in (36). Furthermore, similarly to (26), other identities involving single and double integrals of $Q_{1}$ can be found by rewriting $P_{A}$ in (40) for instance.

\section{CONCLUSION}

In this paper, we have introduced a new demodulation technique for LoRa, based on the detection of possible errors of demodulation. A thorough performance analysis based on order statistics has been derived, in terms of detection rate, SER/BER, and complexity, for both AWGN and Rayleigh channel models. These theoretical results have been validated through simulations. Furthermore, numerical results show that, by adjusting the threshold of the detector, it is possible to asymptotically approach (even for relatively low SNR) the performance of the demodulation at any OSR with the complexity of OSR1. Finally, a discussion introduces different possible applications of the suggested method, therefore paving the way for future works.

\section{APPENDIX A \\ PERIODOGRAM $|Y[k]|^{2}$ FOR $r>1$}

In this appendix, we provide details showing that the periodogram $|Y[k]|^{2}$ for OSR $r>1$ highlights two peaks, such as stated in Section II-A2. Thus, in absence of noise (i.e. $\sigma^{2}=0$ ), the "dechirp" operation in (7), for any $n=0,1, . ., N^{\prime}-1$, leads to

$$
\begin{aligned}
y[n] & =r[n] \exp \left(-2 j \pi \frac{n}{r}\left(\frac{n}{2 N^{\prime}}-\frac{1}{2}\right)\right) \\
& =\exp \left(2 j \pi \frac{n}{r}\left(\frac{m}{N}-u\left(\frac{n}{r}-N+m\right)\right)\right) .
\end{aligned}
$$

The DFT of $y[n]$ in (27), denoted by $Y[k]$, for $k=0,1, . ., N^{\prime}-1$, is given by

$$
\begin{aligned}
& Y[k]=\frac{1}{\sqrt{N^{\prime}}} \sum_{n=0}^{N^{\prime}-1} y[n] e^{-2 j \pi \frac{k n}{N^{\prime}}} \\
& =\frac{1}{\sqrt{N^{\prime}}} \sum_{n=0}^{N^{\prime}-1} \exp \left(2 j \pi \frac{n}{N^{\prime}}\left(m-N u\left(\frac{n}{r}-N+m\right)-k\right)\right) \\
& =\frac{1}{\sqrt{N^{\prime}}} \underbrace{\sum_{n=0}^{r(N-m)-1} \exp \left(2 j \pi \frac{n}{N^{\prime}}(m-k)\right)}_{Y_{A}[k]} \\
& +\underbrace{\sum_{N^{\prime}-1} \exp \left(2 j \pi \frac{n}{N^{\prime}}(m-N-k)\right)}_{\sum_{B}[k]},
\end{aligned}
$$

where $Y_{A}[k]$ and $Y_{B}[k]$ have been defined for clarity purpose. Then by using the sums of geometric series, we obtain:

$$
\begin{aligned}
Y_{A}[k]= & \frac{1-\exp \left(2 j \pi \frac{(m-k)}{N^{\prime}} r(N-m)\right)}{1-\exp \left(2 j \pi \frac{(m-k)}{N^{\prime}}\right)} \\
= & \exp \left(j \pi \frac{(m-k)}{N^{\prime}}(r(N-m)-1)\right) \overbrace{r(N-m)}^{\gamma_{A}} \\
& \times \operatorname{asinc}_{\gamma_{A}}\left(\frac{(m-k)}{N^{\prime}}\right)
\end{aligned}
$$

where asinc $_{\gamma_{A}}$ is the aliased sinc function defined as

$$
\operatorname{asinc}_{\gamma_{A}}(x)=\frac{\sin \left(\pi \gamma_{A} x\right)}{\gamma_{A} \sin (\pi x)} .
$$

Similarly, we find that $Y_{B}[k]$ is expressed as

$$
\begin{aligned}
Y_{B}[k]= & \left.\exp \left(j \pi \frac{(m-N-k)}{N^{\prime}}\left(2 N^{\prime}-r m-1\right)\right)\right) \overbrace{r m}^{\gamma_{B}} \\
& \times \operatorname{asinc}_{\gamma_{B}}\left(\frac{(m-N-k)}{N^{\prime}}\right) .
\end{aligned}
$$


From (29) and (31), we find that $\left|Y_{A}[k]\right|$ is maximum for $k=m$ $\bmod N^{\prime}=m$ (in that case $\left.Y_{B}[m]=0\right)$ and reaches $\left|Y_{A}[m]\right|=$ $r(N-m)$. Similarly we find that $\left|Y_{B}[k]\right|$ is maximum for $k=m-N \bmod N^{\prime}=m-N+N^{\prime}$ (in that case $Y_{A}[m-N+$ $\left.\left.N^{\prime}\right]=0\right)$ and reaches $\left|Y_{B}\left[m-N+N^{\prime}\right]\right|=r m$, finally leading to the expected results, which concludes the proof.

\section{Appendix B}

PROOF OF (15)

Let $X_{0}, X_{1}, \ldots, X_{N-2}$ be $N-1$ iid random variables obeying a $\chi^{2}$ distribution with 2 degrees of freedom, and $Z=\left|\mu+X_{R}+j X_{I}\right|^{2}$ a random variable independent of $X_{k}$, $k=0,1, . ., N-2$, obeying a non-central $\chi^{2}$ distribution with 2 degrees of freedom. Thus, $\mu \in \mathbb{C}$ is defined as a (potentially non-zero) mean, and $\left(X_{R}+j X_{I}\right) \sim \mathcal{N} \mathbb{C}\left(0, \sigma^{2}\right)$ (i.e. the variance of $X_{R}$ and $X_{I}$ is equal to $\left.\frac{\sigma^{2}}{2}\right)$. Using the order statistics of $X_{k}$, i.e. $X_{(0)} \leq X_{(1)} \leq . . \leq X_{(N-2)}$, the probability $\mathbb{P}_{E_{1} \mid h}$, with $0 \leq \beta \leq 1$, corresponds to the probability that at least the largest $X_{k}$ (namely $X_{(N-2)}$ ) is higher than $\beta Z$ while $Z$ is the largest peak, which can be expressed as

$$
\begin{aligned}
P_{E_{1} \mid h} & =\mathbb{P}\left(X_{(N-2)} \geq \beta Z \mid Z \geq X_{(N-2)}\right) \\
& =\frac{\mathbb{P}\left(X_{(N-2)} \geq \beta Z \cap Z \geq X_{(N-2)}\right)}{\mathbb{P}\left(Z \geq X_{(N-2)}\right)} \\
& =\frac{\mathbb{P}\left(Z \geq X_{(N-2)} \geq \beta Z\right)}{\mathbb{P}\left(Z \geq X_{(N-2)}\right)} .
\end{aligned}
$$

It must be noted that the denominator is equal to $1-P_{s \mid h}$ as it corresponds to the probability that the non-central $\chi^{2}$ variable is larger than all the other (centered) $\chi^{2}$ variables. Let us then focus on the numerator of (32). For any $k=0,1, . ., N-2$, the cumulative distribution function (CDF) of the variable $X=\frac{X_{k}}{\sigma^{2}}$ is $F_{X}(x)=1-e^{-x}$, and the $\mathrm{CDF}$ of $\frac{X_{(N-2)}}{\sigma^{2}}$ is $F_{(N-1)}(x)=$ $F_{X}(x)^{N-1}$ (see [29] for details). The distribution of $\frac{X_{(N-2)}}{\sigma^{2}}$ is, in turn, defined as $f_{(N-1)}(x)=\frac{\partial F_{(N-1)}(x)}{x^{2}+y^{2}}$. Furthermore, we denote by $g_{X_{R}, X_{I}}(x, y)=\frac{1}{\pi \sigma^{2}} e^{-\frac{x^{2}+y^{2}}{\sigma^{2}}}$ the bivariate Gaussian distribution of the couple of variables $\left(X_{R}, X_{I}\right)$, where the variance of $X_{R}$ and $X_{I}$ is equal to $\frac{\sigma^{2}}{2}$. Hence, the probability $\mathbb{P}\left(Z \geq X_{(N-2)} \geq \beta Z\right)$ can be expressed as

$$
\begin{aligned}
& \mathbb{P}\left(Z \geq X_{(N-2)} \geq \beta Z\right)=\mathbb{P}\left(\frac{Z}{\sigma^{2}} \geq \frac{X_{(N-2)}}{\sigma^{2}} \geq \frac{\beta Z}{\sigma^{2}}\right) \\
= & \iint_{-\infty}^{+\infty} g_{X_{R}, X_{I}}(x, y) \int_{\frac{z}{\sigma^{2}}}^{\frac{\beta z}{\sigma^{2}}} f_{(N-1)}(u) d u d x d y \\
= & \iint_{-\infty}^{+\infty} g_{X_{R}, X_{I}}(x, y)\left[\left(1-e^{-\frac{z}{\sigma^{2}}}\right)^{N-1}\right. \\
& \left.-\left(1-e^{-\frac{\beta z}{\sigma^{2}}}\right)^{N-1}\right] d x d y .
\end{aligned}
$$

Then, by substituting $z=|\mu+x+j y|^{2}=\left(\mu_{R}+x\right)^{2}+\left(\mu_{I}+y\right)^{2}$, where $\mu_{R}$ (resp. $\mu_{I}$ ) is the real (resp. imaginary) part of $\mu$, and by developing the products in (33) with the binomial formula, we obtain:

$$
\begin{aligned}
& \mathbb{P}\left(Z \geq X_{(N-2)} \geq \beta Z\right) \\
= & \iint_{-\infty}^{+\infty} g_{X_{R}, X_{I}}(x, y) \sum_{k=0}^{N-1}(-1)^{k}\left(\begin{array}{c}
N-1 \\
k
\end{array}\right) \\
& \times\left(e^{-\frac{k|\mu+x+j y|^{2}}{\sigma^{2}}}-e^{-\frac{\beta k|\mu+x+j y|^{2}}{\sigma^{2}}}\right) d x d y \\
= & \frac{1}{\pi \sigma^{2}} \sum_{k=0}^{N-1}(-1)^{k}\left(\begin{array}{c}
N-1 \\
k
\end{array}\right) \\
& \times \iint_{-\infty}^{+\infty} e^{-\frac{1}{\sigma^{2}}\left((k+1) x^{2}+2 k \mu_{R} x+k \mu_{R}^{2}\right)} \\
& e^{-\frac{1}{\sigma^{2}}\left((k+1) y^{2}+2 k \mu_{I} y+k \mu_{I}^{2}\right)} \\
& -e^{-\frac{1}{\sigma^{2}}\left((\beta k+1) x^{2}+2 \beta k \mu_{R} x+\beta k \mu_{R}^{2}\right)} \\
& e^{-\frac{1}{\sigma^{2}}\left((\beta k+1) y^{2}+2 \beta k \mu_{I} y+\beta k \mu_{I}^{2}\right)} d x d y .
\end{aligned}
$$

We remind the following result, as it will be used in some further developments, for any $a, b, c \in \mathbb{R}_{+}^{*}$ :

$$
\int_{-\infty}^{+\infty} \frac{e^{-\frac{1}{\sigma^{2}}\left(\left(a x^{2}+b x+c\right)\right.}}{\sqrt{\pi \sigma^{2}}} d x=\frac{e^{-\frac{b^{2}-4 a c}{4 a \sigma^{2}}}}{\sqrt{a}} .
$$

By substituting $a, b$, and $c$ in (35) by the corresponding values in (34), (34) reduces to:

$$
\begin{aligned}
& \mathbb{P}\left(Z \geq X_{(N-2)} \geq \beta Z\right) \\
= & \sum_{k=0}^{N-1}(-1)^{k}\left(\begin{array}{c}
N-1 \\
k
\end{array}\right)\left(\frac{e^{-\frac{k\left(\mu_{R}^{2}+\mu_{I}^{2}\right)}{(k+1) \sigma^{2}}}}{k+1}-\frac{e^{-\frac{\beta k\left(\mu_{R}^{2}+\mu_{I}^{2}\right)}{(k+1) \sigma^{2}}}}{\beta k+1}\right) .
\end{aligned}
$$

Finally, by substituting $\mu=\sqrt{N^{\prime}} h$, and since $|\mu|^{2}=\mu_{R}^{2}+\mu_{I}^{2}$, then $\mathbb{P}_{E_{1} \mid h}$ in (32) leads to (15), which concludes the proof.

\section{ApPendix C \\ PROOF OF (17)}

We keep the same definitions and assumptions as in Appendix B. However, we here suppose that $X_{(N-2)} \geq Z$, corresponding to the case where a demodulation error occurs. Then, the probability $\mathbb{P}_{E_{0} \mid h}$ is expressed as

$$
\begin{aligned}
P_{E_{0} \mid h} & =\mathbb{P}\left(X \geq \beta X_{(N-2)} \mid X_{(N-2)} \geq Z\right) \\
& =\frac{\mathbb{P}\left(\frac{1}{\beta} X \geq X_{(N-2)} \cap X_{(N-2)} \geq Z\right)}{\mathbb{P}\left(X_{(N-2)} \geq Z\right)},
\end{aligned}
$$

where $X=Z$ or $X=X_{(N-3)}$. It must be noted that the denominator is equal to $P_{s \mid h}$ as it corresponds to the probability that the non-central $\chi^{2}$ variable is lower than at least the largest (centered) $\chi^{2}$ variable $X_{(N-2)}$. To develop the numerator in (37), one can notice that either we have $X_{(N-2)} \geq X=Z \geq$ $X_{(N-3)}$ and in that case $\frac{1}{\beta} Z \geq X_{(N-2)} \geq Z \geq X_{(N-3)}$, or $X_{(N-2)} \geq X=X_{(N-3)} \geq Z$ and in that case $\frac{1}{\beta} X_{(N-3)} \geq$ $X_{(N-2)} \geq X_{(N-3)} \geq Z$. Since both events are disjoints, then the numerator in (37) can be developed as 


$$
\begin{aligned}
& \mathbb{P}\left(\frac{1}{\beta} X \geq X_{(N-2)} \cap X_{(N-2)} \geq Z\right)= \\
& \underbrace{\mathbb{P}\left(\frac{1}{\beta} Z \geq X_{(N-2)} \geq Z \geq X_{(N-3)}\right)}_{P_{A}} \\
& +\underbrace{\mathbb{P}\left(\frac{1}{\beta} X_{(N-3)} \geq X_{(N-2)} \geq X_{(N-3)} \geq Z\right)}_{P_{B}},
\end{aligned}
$$

where $P_{A}$ and $P_{B}$ have been defined for clarity matter. We denote by $f_{(N-3),(N-2)}(u, v), 0 \leq u<v<+\infty$, the joint distribution of $\frac{X_{(N-2)}}{\sigma^{2}}$ and $\frac{X_{(N-3)}}{\sigma^{2}}$, then from [29] we get

$$
f_{(N-3),(N-2)}(u, v)=(N-1)(N-2) e^{-u}\left(1-e^{-u}\right)^{N-3} e^{-v} .
$$

We can express $P_{A}$ using (39) as

$$
\begin{aligned}
P_{A}= & \mathbb{P}\left(\frac{Z}{\beta \sigma^{2}} \geq \frac{X_{(N-2)}}{\sigma^{2}} \geq \frac{Z}{\sigma^{2}} \geq \frac{X_{(N-3)}}{\sigma^{2}}\right) \\
= & \iint_{-\infty}^{+\infty} g_{X_{R}, X_{I}}(x, y) \\
& \times \int_{0}^{\frac{z}{\sigma^{2}}} \int_{\frac{z}{\sigma^{2}}}^{\frac{z}{\beta \sigma^{2}}} f_{(N-3),(N-2)}(u, v) d v d u d x d y \\
= & (N-1)(N-2) \iint_{-\infty}^{+\infty} g_{X_{R}, X_{I}}(x, y)\left(e^{-\frac{z}{\sigma^{2}}}-e^{-\frac{z}{\beta \sigma^{2}}}\right) \\
& \times \int_{0}^{\frac{z}{\sigma^{2}}} e^{-u}\left(1-e^{-u}\right)^{N-3} d u d x d y \\
= & (N-1)(N-2) \iint_{-\infty}^{+\infty} g_{X_{R}, X_{I}}(x, y)\left(e^{-\frac{z}{\sigma^{2}}}-e^{-\frac{z}{\beta \sigma^{2}}}\right) \\
& \times \int_{0}^{\frac{z}{\sigma^{2}}} \sum_{k=0}^{N-3}(-1)^{k}\left(\begin{array}{c}
N-3 \\
k
\end{array}\right) e^{-(k+1) u} d u d x d y \\
= & (N-1)(N-2) \iint_{-\infty}^{+\infty} g_{X_{R}, X_{I}}(x, y)\left(e^{-\frac{z}{\sigma^{2}}}-e^{-\frac{z}{\beta \sigma^{2}}}\right) \\
& \times \sum_{k=0}^{N-3}(-1)^{k}\left(\begin{array}{c}
N-3 \\
k
\end{array}\right) \frac{\left(1-e^{-\frac{(k+1) z}{\sigma^{2}}}\right)}{k+1} d x d y .
\end{aligned}
$$

Then, since we have

$$
\sum_{k=0}^{N-3} \frac{(-1)^{k}\left(\begin{array}{c}
N-3 \\
k
\end{array}\right)}{k+1}=\frac{1}{N-2},
$$

and by using the result in (35), (40) leads to

$$
\begin{aligned}
P_{A} & =(N-1)(N-2)\left[\frac{1}{N-2}\left(\frac{1}{2} e^{-\frac{\mu^{2}}{2 \sigma^{2}}}-\frac{\beta}{\beta+1} e^{-\frac{\mu^{2}}{(\beta+1) \sigma^{2}}}\right)\right. \\
& \left.-\sum_{k=0}^{N-3} \frac{(-1)^{k}\left(\begin{array}{c}
N-3 \\
k
\end{array}\right)}{k+1}\left(\frac{\left(e^{-\frac{(k+2) \mu^{2}}{(k+3) \sigma^{2}}}\right)}{k+3}-\frac{\left(e^{-\frac{\left(k+1+\frac{1}{\beta}\right) \mu^{2}}{\left(k+2+\frac{1}{\beta}\right) \sigma^{2}}}\right)}{k+2+\frac{1}{\beta}}\right)\right] .
\end{aligned}
$$

Similarly to the developments leading to $P_{A}$, we obtain $P_{B}$ from (38) as

$$
\begin{aligned}
P_{B}= & \mathbb{P}\left(\frac{X_{(N-3)}}{\beta \sigma^{2}} \geq \frac{X_{(N-2)}}{\sigma^{2}} \geq \frac{X_{(N-3)}}{\sigma^{2}} \geq \frac{Z}{\sigma^{2}}\right) \\
= & \iint_{-\infty}^{+\infty} g_{X_{R}, X_{I}}(x, y) \\
& \times \int_{\frac{z}{\sigma^{2}}}^{+\infty} \int_{u}^{\frac{u}{\beta}} f_{(N-3),(N-2)}(u, v) d v d u d x d y \\
= & (N-1)(N-2) \iint_{-\infty}^{+\infty} g_{X_{R}, X_{I}}(x, y) \\
& \times \int_{\frac{z}{\sigma^{2}}}^{+\infty}\left(e^{-u}-e^{-\frac{u}{\beta}}\right) \\
& \times \sum_{k=0}^{N-3}(-1)^{k}\left(\begin{array}{c}
N-3 \\
k
\end{array}\right) e^{-(k+1) u} d u d x d y \\
= & (N-1)(N-2) \iint_{-\infty}^{+\infty} g_{X_{R}, X_{I}}(x, y) \\
& \times \sum_{k=0}^{N-3}(-1)^{k}\left(\begin{array}{c}
N-3 \\
k
\end{array}\right) \\
& \times\left(\frac{e^{-\frac{(k+2) z}{\sigma^{2}}}}{k+2}-\frac{e^{-\frac{\left(k+1+\frac{1}{\beta}\right) z}{\sigma^{2}}}}{k+1+\frac{1}{\beta}}\right) d x d y \\
= & (N-1)(N-2) \sum_{k=0}^{N-3}(-1)^{k}\left(\begin{array}{c}
N-3 \\
k
\end{array}\right) \\
& \times\left(\frac{e^{-\frac{(k+2) \mu^{2}}{(k+3) \sigma^{2}}}}{(k+2)(k+3)} \frac{e^{-\frac{\left(k+1+\frac{1}{\beta}\right) \mu^{2}}{\left(k+2+\frac{1}{\beta}\right) \sigma^{2}}}}{\left(k+1+\frac{1}{\beta}\right)\left(k+2+\frac{1}{\beta}\right)}\right) .
\end{aligned}
$$

Finally, by substituting $\mu=\sqrt{N^{\prime}} h$, and since $|\mu|^{2}=\mu_{R}^{2}+\mu_{I}^{2}$, then $\mathbb{P}_{E_{0} \mid h}$ leads to (17), which concludes the proof.

\section{ACKNOWLEDGMENT}

The author would like to thank Dr Basile De Loynes from ENSAI, Rennes, France, for his insightful remarks and advice about order statistics.

\section{REFERENCES}

[1] G. A. Akpakwu, B. J. Silva, G. P. Hancke, and A. M. Abu-Mahfouz, "A Survey on 5G Networks for the Internet of Things: Communication Technologies and Challenges," IEEE Access, vol. 6, no. 12, pp. 3619 - 3647, December 2017.

[2] W. Ayoub, A. Samhat, F. Nouvel, M. Mroue, and J.-C. Prévotet, "Internet of Mobile Things: Overview of LoRaWAN, DASH7, and NB-IoT in LPWANs standards andSupported Mobility," in proc. of ICT'18, June 2018, pp. $1561-1581$.

[3] K. Mekki, E. Bajic, F. Chaxel, and F. Meyer, "A comparative study of LPWAN technologies for large-scale IoT deployment," ICT Express, vol. 5, no. 1, pp. $1-7$, March 2019.

[4] "Lorawan ${ }^{\mathrm{TM}}$ specification," LoRa Alliance Technical Committee, October 2017, ver. 1.1.

[5] M. Winkler, "Chirp signals for communications," IEEE WESCON Convention Record, p. 7, 1962.

[6] L. Vangelista, "Frequency Shift Chirp Modulation: The LoRa Modulation," IEEE Signal Processing Letters, vol. 24, no. 12, pp. 1818 - 1821, December 2017. 
[7] M. Chiani and A. Elzanaty, "On the LoRa Modulation for IoT: Waveform Properties and Spectral Analysis," IEEE Internet of Things Journal, vol. 6 , no. 5, pp. 8463-8470, October 2019.

[8] H. Tanaka, "A frequency and timing synchronization circuit making use of a chirp signal," EU Patent 99250 131.2, 1998.

[9] M. Xhonneux, D. Bol, and J. Louveaux, "A Low-complexity Synchronization Scheme for LoRa End Nodes," ArXiv, no. arXiv:1912.11344, pp. 1 - 10, December 2019.

[10] R. Ghanaatian, O. Afisiadis, M. Cotting, and A. Burg, "Lora Digital Receiver Analysis and Implementation," in proc. of ICASSP'19, Brighton, United Kingdom, May 2019, pp. 1498-1502.

[11] C. Bernier, F. Dehmas, and N. Deparis, "Low Complexity LoRa Frame Synchronization for Ultra-Low Power Software-Defined Radios," IEEE Transactions on Communications, vol. 68, no. 5, pp. 3140-3152, May 2020.

[12] A. Marquet and N. Montavont, "Carrier and Symbol Synchronisation for LoRa Receivers," in proc. of EWSN'20, Lyon, France, February 2020 , pp. $1-6$.

[13] V. Savaux, C. Delacourt, and P. Savelli, "On Time-Frequency Synchronization in LoRa System: From Analysis to Near-Optimal Algorithm," TechRxiv, pp. 1 - 11, January 2021. [Online]. Available: https://www. techrxiv.org/articles/preprint/On_Time-Frequency_Synchronization_in_ LoRa_System_From_Analysis_to_Near-Optimal_Algorithm/13560158

[14] M. A. Ben Temim, G. Ferré, B. Laporte-Fauret, D. Dallet, B. Minger, and L. Fuché, "An enhanced receiver to decode superposed lora-like signals," IEEE Internet of Things Journal, vol. 7, no. 8, pp. 7419-7431, August 2020.

[15] V. Savaux and P. Savelli, "Frequency Domain Preamble-Based Channel Estimation and Equalization in LoRa," International Journal of Mobile Network Design and Innovation, pp. 1 - 8, 2021.

[16] G. Ferré and A. Giremus, "LoRa Physical Layer Principle and Performance Analysis," in proc. of IEEE International Conference on Electronics, Circuits and Systems 2018 (ICECS'18). Bordeaux, France: IEEE, 12 2018, pp. $1-4$

[17] T. Elshabrawy and J. Robert, "Closed-Form Approximation of LoRa Modulation BER Performance," IEEE Communications Letters, vol. 22, no. 9, pp. 1778 - 1781, September 2018.

[18] J. Proakis and M. Salehi, Digital Communications. McGraw-Hill, 2008

[19] C. F. Dias, E. R. de Lima, and G. Fraidenraich, "Bit Error Rate Closed-Form Expressions for LoRa Systems under Nakagami and Rice Fading Channels," Sensors, vol. 19, no. 20, pp. 1 - 11, October 2019.

[20] V. Savaux and G. Ferré, "Simple Asymptotic BER Expressions for LoRa System over Rice and Rayleigh Channels," in proc. of WTS'21, San Fransisco, CA, USA, April 2021, pp. 1 - 5.

[21] M. J. Faber, K. M. van der Zwaag, W. G. V. dos Santos, H. R. d. O. Rocha, M. E. V. Segatto, and J. A. L. Silva, "A Theoretical and Experimental Evaluation on the Performance of LoRa Technology," IEEE Sensors Journal, vol. 20, no. 16, pp. 9480-9489, August 2020.

[22] V. Savaux, C. Delacourt, and P. Savelli, "Considering Sync Word and Header Error Rates for Performance Assessment in LoRa System," in proc. of WTS'21, San Fransisco, CA, USA, April 2021, pp. 1 - 5.

[23] O. Afisiadis, M. Cotting, A. Burg, and A. Balatsoukas-Stimming, "On the Error Rate of the LoRa Modulation with Interference," IEEE Transactions on Wireless Communications, vol. 19, no. 2, pp. 1292 - 1304, February 2020.

[24] A. Marquet, N. Montavont, and G. Z. Papadopoulos, "Towards an sdr implementation of lora: Reverse-engineering, demodulation strategies and assessment over rayleigh channel," Computer Communications, vol. 153, pp. 595-605, March 2020.

[25] C. Delacourt, P. Savelli, and V. Savaux, "A Cloud RAN Architecture for LoRa," URSI Radio Science Letters, vol. 2, pp. 1 - 5, December 2020

[26] H. A. David and H. N. Nagaraja, Order Statistics. J. Wiley \& Sons, 2003.

[27] K. U. Wannenwetsch, "Deterministic Sparse FFT Algorithms," Ph.D. dissertation, Georg-August-Universität Göttingen, 2016.

[28] A. Nuttall, "Some integrals involving the $Q_{M}$ function," IEEE Transactions on Information Theory, vol. 21, no. 1, pp. 95-96, 1975.

[29] H. A. David and H. N. Nagaraja, Order Statistics. Hoboken, NJ: J. Wiley \& Sons, 2003, ch. 2: Basic Distribution Theory, pp. 9 - 32.

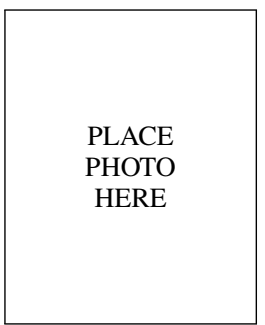

Vincent Savaux (M'16) received his engineering degree from the high School of Engineering, ECAM Rennes, an M.Sc. degree from the University of Rennes 1, France, in 2010, and his Ph.D. degree in telecommunications from École Supérieur d'ÉlectricitéSupélec in 2013. From 2014 to 2015, he was a postdoctoral researcher with the Signal, Communication, and Embedded Electronics (SCEE) Research Group, CentraleSupélec, Campus de Rennes, France. Since 2016, he has been a research engineer with the Institute of Research and Technology $b<>$ com, Rennes, France. His research activities mainly focus on signal processing for wireless systems, including IoT technologies such as NB-IoT and LoRa. He received the 2014 Exemplary Reviewer Appreciation from the IEEE WIRELESS COMMUNICATIONS LETTERS and the IEEE COMMUNICATIONS LETTERS 